\section{CPS-085 STEWARDSHIPS OF HEPATITIS C VIRUS PATIENTS IN PRISONS}

${ }^{1} M D$ Gil-Sierra*, ${ }^{2} F$ Tellez-Perez, ${ }^{1} E$ Rios-Sanchez, ${ }^{1} M D P$ Briceño-Casado, ${ }^{1} S$ FenixCaballero, ${ }^{1} \mathrm{C}$ Martinez-Diaz, ${ }^{1} \mathrm{E}$ J Alegre-Del Rey, ${ }^{1} \mathrm{C}$ Palomo-Palomo, ${ }^{1} \mathrm{~J}$ Diaz-Navarro, ${ }^{1} \mathrm{JF}$ Lopez-Vallejo, 'JM Borrero-Rubio. 'Hospital Universitario de Puerto Real, Pharmacy, Puerto Real, Spain; ${ }^{2}$ Hospital Universitario de Puerto Real, Internal Medicine Service, Puerto Real, Spain

\subsection{6/ejhpharm-2019-eahpconf.234}

Background To eliminate hepatitis $\mathrm{C}$ virus (HCV) infection in institutionalised patients it is necessary to reduce the risk of transmission in the general population. There is a high prevalence of HCV infection in the prison population (PP).

Purpose To develop a multidisciplinary programme for the diagnosis and treatment of HCV infection in PP of three prisons and evaluate the effectiveness and safety of treatment for $\mathrm{HCV}$.

Material and methods A multidisciplinary programme (prison physicians, hospital pharmacists and physicians of infectious diseases) from June 2016 to September 2018 was designed. Total PP of three penitentiary centres were included. HCV serology tests were made. PP with $\mathrm{HCV}+$ serology were analysed: HCV genotype and hepatic fibrosis stage. Medical assistance and drug dispensing were performed twice a week. At first, HCV-PP with higher hepatic fibrosis stage (F4-F3) were treated. For the past year, all HCV-PP received therapy regardless of hepatic fibrosis stage. The following variables were collected: gender, patient type (naïve/pretreated), HIV coinfection, therapy, withdrawal treatments and HCV recurrence. Effectiveness end points were end of treatment response (EOT) and sustained virologic response at week 12 (SVR12). EOT was determined as undetectable HCV-RNA at treatment completion and SVR12 as undetectable HCV-RNA 12 weeks after the ending of treatment. Security was evaluated based on related adverse effects (AE) and deaths.

Results HCV serology tests of 2068 patients were made: 181 patients were $\mathrm{HCV}$ +and treated. Variables data: 125 (69.1\%) males, 157 (86.7\%) naive, 49 (27.1\%) HIV/HCV coinfected. Hepatic fibrosis stages were: 50 (27.6\%) F4, 28 (15.5\%) F3, $34(18.8 \%)$ F2 and $69(38.1 \%)$ F0-1. HCV genotype: 59 (32.6\%) G1a, 33 (18.3\%) G1b, two (1.1\%) G2, 50 (27.6\%) G3, $35(19.3 \%)$ G4 and two (1.1\%) G-unclear. The most frequent treatments were: 51 (28,2\%) glecaprevir/pibrentasvir, 41 (22.7\%) sofosbuvir/velpatasvir, 29 (16\%) elbasvir/grazoprevir and $25(13.8 \%)$ sofosbuvir/ledipasvir. One-hundred and eighteen $(65.2 \%)$ patients were treated for 12 weeks. There were five $(2.8 \%)$ withdrawal treatments. HCV recurrence: four (2.2\%) patients. One-hundred and forty-three completed treatment and 114 had measurable SVR12 at the end of the study: 136/143 (95.1\%) achieved EOT and 103/114 (90.4\%) SVR12. $\mathrm{AE}$ was one $(0.5 \%)$ hepatic decompensation, which caused death.

Conclusion The multidisciplinary programme diagnosed and treated all PP with HCV infection, although some withdrawal treatments were recorded. EOT and SVR12 were achieved in most patients. An AE leading a death.

\section{REFERENCES AND/OR ACKNOWLEDGEMENTS}

None.

No conflict of interest.

\section{CPS-086 A FULLY INTEGRATED CLINICAL TRIAL-LIKE SYSTEM TO MANAGE AND MONITOR PERSISTENCE IN PLANNED HEPATITIS C TREATMENT}

${ }^{1} \mathrm{G}$ Gomes*, ${ }^{1} \mathrm{~A}$ Figueira, ${ }^{1} \mathrm{C}$ Sequeira, ${ }^{1} \mathrm{~S}$ Ferreira da Silva, ${ }^{1} \mathrm{~F}$ Machado, ${ }^{2} \mathrm{P}$ Figueiredo, ${ }^{3}$ E Rabadão, ${ }^{4} V$ Andreozzi, ${ }^{5} \mathrm{~J}$ Félix, ${ }^{1} \mathrm{~J}$ Feio. ${ }^{1}$ Centro Hospitalar e Universitário de Coimbra, Hospital Pharmacy, Coimbra, Portugal; ${ }^{2}$ Centro Hospitalar e Universitário de Coimbra, Gastroenterology Department, Coimbra, Portugal; ${ }^{3}$ Centro Hospitalar e Universitário de Coimbra, Infectious Disease Department, Coimbra, Portugal; ${ }^{4}$ Exigo Consultores, Quantitative Methods, Lisbon, Portugal; ${ }^{5}$ Exigo Consultores, Administration, Lisbon, Portugal

\subsection{6/ejhpharm-2019-eahpconf.235}

Background Portugal was one of the first countries in the world to have a universal access programme to new direct-acting antivirals (DAA) therapy for hepatitis C. The implementation of such a policy in our university hospital was managed by the hospital pharmacy based on a new and specific system designated fully integrated clinical trials-like system (CTLIKE), allowing full traceability of hepatitis $\mathrm{C}$ therapy and patient outcomes.

Purpose Our aim was to assess CTLIKE system efficiency in terms of patients' persistence on DAA therapy for hepatitis C in our hospital.

Material and methods CTLIKE is based on a set of day-to-day routines and protocols, supported by a dedicated software with the aim of controlling DAA dispensing and refiling, and also therapy and patient outcomes monitoring, with the ultimate goal of capturing full benefits from hepatitis $\mathrm{C}$ treatment for all stakeholders involved. CTLIKE is managed by the hospital pharmacy in our university hospital. The efficiency of CTLIKE was assessed by measuring persistence, defined as remaining in therapy and not discontinuing (end of treatment). The Kaplan-Meier method was used for crude survival calculations. The risk of DAA treatment discontinuation was estimated by Cox proportional hazard models. Adherence was a secondary exploratory endpoint calculated by the pill count method.

Results Data supporting this research was retrospectively collected and refers to 721 patients initiating DAA therapy since January 2015. Mean (SD) age at therapy initiation was 49.9 (10.8) years and $76.0 \%$ were male: genotype 1 (70.8\%), metavir F1 (33.3\%) and treatment naïve (69.5\%). The vast majority of treatment regimens were sofosbuvir-based (94.7\%). Programmed treatment duration was: 12 weeks (73.6\%) and 24 weeks (26.4\%). Premature treatment discontinuation before the planned 12 and 24 weeks was estimated at $9.5 \%$ (95\% CI: $6.9 \%$ to $12.1 \%)$ and $20.4 \%$ (95\% CI: $14.4 \%$ to $26.0 \%$ ), respectively. Non-cirrhotic patients (HR discontinuation $=0.73,95 \% \mathrm{CI}: 0.57$ to 0.95$)$ and males $(\mathrm{HR}=0.85,95 \% \mathrm{CI}: 0.69$ to 1.04$)$ were more likely to persist in treatment. Adherence level $\geq 95 \%$ to DAA treatment (pill count) occurred in $97.8 \%$ and $98.9 \%$ of the 12 weeks and 24 weeks subgroups.

Conclusion The CTLIKE system revealed full efficacy in DAA dispensing and hepatitis $\mathrm{C}$ treatment outcomes monitoring, guaranteeing very high persistence and adherence rates in hepatitis $\mathrm{C}$ therapy in this real-world setting.

\section{REFERENCES AND/OR ACKNOWLEDGEMENTS}

None.

No conflict of interest. 